AIP Applied Physics

\title{
On the method of photoluminescence spectral intensity ratio imaging of silicon bricks: Advances and limitations
}

Bernhard Mitchell, Jürgen W. Weber, Daniel Walter, Daniel Macdonald, and Thorsten Trupke

Citation: J. Appl. Phys. 112, 063116 (2012); doi: 10.1063/1.4752409

View online: http://dx.doi.org/10.1063/1.4752409

View Table of Contents: http://jap.aip.org/resource/1/JAPIAU/v112/i6

Published by the American Institute of Physics.

\section{Related Articles}

Investigation of the thermal charge "trapping-detrapping" in silicon nanocrystals: Correlation of the optical properties with complex impedance spectra

Appl. Phys. Lett. 101, 242108 (2012)

Inhomogeneous linewidth broadening and radiative lifetime dispersion of size dependent direct bandgap radiation in Si quantum dot

AlP Advances 2, 042162 (2012)

Calibration of the photoluminescence technique for measuring concentrations of shallow dopants in $\mathrm{Ge}$

J. Appl. Phys. 112, 103701 (2012)

On the origin of inter band gap radiative emission in crystalline silicon

AlP Advances 2, 042135 (2012)

Electronic states and curved surface effect of silicon quantum dots

Appl. Phys. Lett. 101, 171601 (2012)

\section{Additional information on J. Appl. Phys.}

Journal Homepage: http://jap.aip.org/

Journal Information: http://jap.aip.org/about/about_the_journal

Top downloads: http://jap.aip.org/features/most_downloaded

Information for Authors: http://jap.aip.org/authors

\section{ADVERTISEMENT}

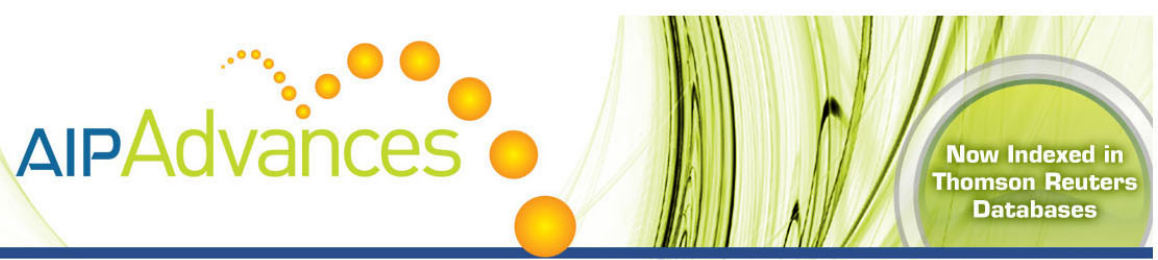

\section{Explore AIP's open access journal: •Rapid publication \\ - Article-level metrics \\ Post-publication rating and commenting}




\title{
On the method of photoluminescence spectral intensity ratio imaging of silicon bricks: Advances and limitations
}

\author{
Bernhard Mitchell, ${ }^{1, a)}$ Jürgen W. Weber, ${ }^{2}$ Daniel Walter, ${ }^{3}$ Daniel Macdonald, ${ }^{3}$ \\ and Thorsten Trupke ${ }^{1,2}$ \\ ${ }^{1}$ School of Photovoltaic and Renewable Energy Engineering, University of New South Wales, Sydney, \\ NSW 2052, Australia \\ ${ }^{2}$ BT Imaging, 1 Blackburn St, Surry Hills, NSW 2010, Australia \\ ${ }^{3}$ Research School of Engineering, College of Engineering and Computer Science, The Australian National \\ University, Canberra, ACT 0200, Australia
}

(Received 19 March 2012; accepted 9 August 2012; published online 24 September 2012)

\begin{abstract}
Spectral photoluminescence imaging is able to provide quantitative bulk lifetime and doping images if applied on silicon bricks or thick silicon wafers. A comprehensive study of this new method addresses previously reported artefacts in low lifetime regions and provides a more complete understanding of the technique. Spectrally resolved photoluminescence measurements show that luminescence originating from sub band gap defects does not cause those artefacts. Rather, we find that optical light spreading within the silicon CCD is responsible for most of the distortion in image contrast and introduce a method to measure and remove this spreading via image deconvolution. Alternatively, image blur can be reduced systematically by using an InGaAs camera. Results of modelling this alternative camera type and experiments are shown and discussed in comparison. In addition to eliminating the blur effects, we find a superior accuracy for lifetimes above $100 \mu$ s with significantly shorter, but dark noise limited exposure times. (C) 2012 American Institute of Physics. [http://dx.doi.org/10.1063/1.4752409]
\end{abstract}

\section{INTRODUCTION}

A new quantitative photoluminescence (PL) imaging based method to measure spatially resolved bulk lifetimes and doping on silicon bricks has been reported recently. ${ }^{1}$ Two PL images are taken with different spectral filters mounted in front of the camera lens and are used to determine a spectral intensity ratio image which can be converted into a bulk lifetime image by pure one dimensional modelling. The method is a specific application of photoluminescence imaging of silicon, ${ }^{2}$ which extends the analysis from a direct relative intensity to a relative spectral intensity ratio. ${ }^{3-7}$ Other recent developments used the time dependence of the luminescence radiation as an alternative approach to quantitatively analyse the lifetime, ${ }^{8,9}$ which could potentially be applied to bricks, but will be somewhat limited by noise in low lifetime areas.

In extension to the global lifetime information being able to extract by direct imaging methods like the one described in this paper, local microscopic lifetime information is of high value for the development of solar cell device structures and also for research into the electrical properties of grain boundaries and other structural defects. A micro PL spectroscopy lifetime mapping as demonstrated by Gundel et $a l^{10}$ is a promising development and can potentially be applied to silicon bricks for the investigation of grain boundaries and their decoration with metals and other impurities.

To date, calibrated lifetime measurements on multicrystalline silicon bricks or thick silicon wafers are commonly performed using either the photoluminescence or photoconductance (PC) signal, either in transient or quasi-

\footnotetext{
${ }^{\text {a) }}$ Author to whom correspondence should be addressed. Electronic mail: bernhard.mitchell@unsw.edu.au.
}

steady-state mode. ${ }^{11-15}$ Point measurements, line scans, or low resolution pixel maps can be taken with these techniques. In terms of brick measurements, microwave detected photoconductance decay (MWPCD) provides an effective lifetime map, and quasi steady state photoconductance (QSSPC) detected maps can give actual bulk lifetime via the calculation of an average injection level. ${ }^{13} \mathrm{~A}$ detailed analysis of the measurement of bulk lifetimes from QSSPC data on silicon bricks was recently presented. ${ }^{15}$

In this work we will report on improvements and new findings in the context of the method of using photoluminescence spectral intensity ratios for bulk lifetime imaging on silicon bricks. The basic underlying method and proof of concept were presented by Mitchell et al., ${ }^{1}$ reporting on significant experimental artefacts, particularly in impurity rich low lifetime regions near the top and bottom of bricks. The cause and the metrics of these artefacts is analysed in this paper. Optical deconvolution is shown to be able to largely remove the effect of light spreading in the detection unit, while comparable measurements using an InGaAs camera are confirmed to be unaffected by light spreading. We discuss the quantitative accuracy and noise levels of both cameras when applied to the photoluminescence intensity ratio (PLIR) method.

\section{THEORY AND METHODS}

The theory of the method and its application to silicon bricks has been discussed previously ${ }^{1}$ and is based on the PLIR method, first introduced for measuring diffusion lengths on silicon solar cells by Würfel et al. ${ }^{3}$ Figure 1 illustrates the basic principle of the method as the luminescence emission spectrum of the sample shifts on the short wavelength flank as the bulk lifetime increases. This is fundamentally reasoned 
in the decay of the silicon absorption coefficient within the wavelength range of the emission. Measurable changes in the integrating imaging application are limited to the range of about $1000-1075 \mathrm{~nm}$.

\section{Modelling emission}

Recently, a simplification of the modelling of the luminescence emission spectra has been achieved by Green. ${ }^{16}$ Previous theoretical modelling of the brick photoluminescence intensity ratio to bulk lifetime transfer function used analytical expressions for the depth dependent excess carrier density. The calculation of the total detected intensity involved two numerical integrations: one as a function of sample position (depth) and a second one as a function of wavelength. Green ${ }^{16}$ presented an analytical solution of the spectral composition of the band-to-band luminescence for monochromatic illumination as a function of the diffusion length, avoiding the spatial numerical integration

$$
\left.P L\left(\tau_{b}\right)\right|_{S \rightarrow \infty} \propto \int_{\lambda_{0}}^{\lambda} r_{s p}(\lambda) \frac{\alpha_{b b}\left(\lambda_{\text {laser }}\right) L^{2}\left(\tau_{b}\right)}{\left[\alpha\left(\lambda_{\text {laser }}\right)+\alpha(\lambda)\right]\left[1+\alpha\left(\lambda_{\text {laser }}\right) L\left(\tau_{b}\right)\right]\left[1+\alpha(\lambda) L\left(\tau_{b}\right)\right]} \Theta(\lambda) d \lambda,
$$

where $S$ is the surface recombination velocity, $r_{\text {sp }}$ the spontaneous emission, $\alpha_{\mathrm{bb}}$ the band-to-band, and $\alpha$ the total silicon absorption coefficient, $L$ the diffusion length, and $\Theta$ the spectral sensitivity of the detection system including filters, lens, and CCD. The analytical integral is an elegant way to determine the integral luminescence intensity, since the minority carrier distribution and the luminescence reabsorption is implicitly accounted for. The only remaining parameters $\alpha, L$, and $\Theta$ are all temperature dependent as is accounted for in the modelling. Temperature dependent data for the silicon absorption coefficient are taken from Green. ${ }^{17}$

Comparison of the analytical solution and the numerical integration of the depth integral showed that both approaches reveal identical results. We use the analytical solution of Eq. (1) for all calculations in this study.

\section{Defect luminescence}

Impurities such as transition metals, oxygen, or carbon introduce multiple intra band gap levels in silicon and thus

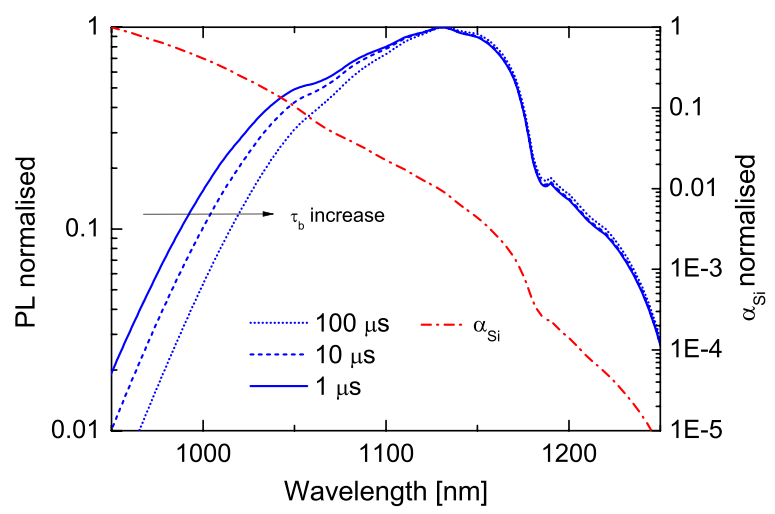

FIG. 1. Calculated silicon brick luminescence emission spectra for 1, 10, and $100 \mu \mathrm{s}$ bulk lifetime and band-to-band silicon absorption coefficient and as a function of wavelength both normalised to the maximum and plotted semi-logarithmically. The illustrated luminescence spectrum is emitted by an infinitely thick silicon sample with infinite surface recombination velocity when excited with a $900 \mathrm{~nm}$ laser. Changes in the emission spectrum caused by bulk lifetime variations are indicated. severely reduce the minority carrier lifetime, especially if the impurity levels are located near the middle of the band gap. ${ }^{18}$ The levels of interstitial iron or FeB pairs, both prominent impurities in cast silicon bricks, are well known and have recently been proposed to be responsible for PL peaks around $1300 \mathrm{~nm},{ }^{19,20}$ hence potentially affecting the silicon detected spectral intensity ratios.

Additionally, the so-called D bands are detectable at reduced temperatures in crystalline silicon, with the D1 band often being detectable as a broad spectral peak with a centre wavelength of about $1550 \mathrm{~nm}^{21-24}$. Recent work found indications that these lines originate from dislocation clusters decorated with metal and oxygen precipitates. ${ }^{25-27}$ In earlier work, such long wavelength defect luminescence was suspected to be a possible explanation for substantially overestimated bulk lifetime values, particularly in the impurity rich top- and bottom regions of bricks. ${ }^{20,28}$ Since light of $1550 \mathrm{~nm}$ wavelength cannot be detected by a silicon CCD, D bands can be ruled out as a cause of the observed artefacts, but could affect the ratios measured with an InGaAs camera.

We use room-temperature spectral photoluminescence measurements to obtain clear experimental evidence if there is defect luminescence contributing to the long pass filtered PL image, thus distorting the ratio. A fibre-coupled laser diode with $880 \mathrm{~nm}$ centre wavelength and a maximum optical power output of $75 \mathrm{~W}$ was used as an excitation source for both spectral and imaging experiments. For the spectral measurements we use lower power settings since the laser beam is collimated by a lens of $50 \mathrm{~mm}$ focal length resulting in a circularly illuminated spot of $1.2 \mathrm{~cm}$ in diameter. This size is sufficient to avoid lateral minority carrier current flow in the central measurement spot, which would thus be affecting the measurement. The injection level of the illumination spot is enhanced compared to the level in the imaging application as the illumination intensity is chosen at about 20 suns $\left(2 \mathrm{~W} / \mathrm{cm}^{2}\right)$ to reach sufficiently strong signal. However, this elevated injection level does not corrupt the assessment since the spectral composition of the luminescence spectra is not significantly injection dependent at room temperature, particularly not between 1100 and $1300 \mathrm{~nm}$. The spectra are 
measured by passing the luminescence from various silicon bricks through an Oriel Ms260i 1/4 m monochromator with a grating blazed at $800 \mathrm{~nm}$. A liquid nitrogen cooled $\mathrm{InGaAs}$ photodiode with an active area of $1 \mathrm{~mm}^{2}$ and a build-in low noise preamplifier with a transimpedance of $10^{9} \mathrm{~V} / \mathrm{A}$ serves as detector. The signal is modulated at $30 \mathrm{~Hz}$ and measured by a Stanford Research SR 830 lock-in amplifier.

\section{Optical distortions and image deconvolution}

Optical imaging applications are affected by a number of image artefacts. Since silicon is a weak emitter of bandto-band luminescence, ${ }^{29}$ small light intensities need to be detected reliably and ideally with high signal to noise ratio. A low f-number lens helps increase the signal to noise ratio. However, low f-numbers are generally associated with more optical aberration. Another potential source of optical image artefacts is the silicon CCD itself. Due to the long penetration length of the silicon luminescence signal within silicon, there is an enhanced probability of light being detected inside a pixel of the CCD after significant lateral scattering within the CCD has occurred. These non-idealities can be described by a so-called point spread function (PSF), which describes how an ideal point source is detected by the imaging system, which can be used partially reverse the light spread via deconvolution. And since silicon luminescence is measured with a silicon detector at its band-edge the point spread effects are largely wavelength dependent.

We propose a method to determine the point spread functions for both short and long pass filter detection. Silicon bricks are an ideal test case for such studies, because wafers and cells are subject to convolution effects from both light scattering (texture, internal reflection) and lateral diffusion effects within the sample itself. The latter can be neglected for the silicon bricks investigated, which are $156 \mathrm{~mm}$ thick and have polished surfaces.

To be able to correct point spreading artefacts as accurately as possible, the PSF must be determined for a dynamic range covering at least seven orders of magnitude in image intensity, since, if measuring with full resolution of $1024 \times 1024$ pixel, more than $1 \times 10^{6}$ pixels can contribute to the intensity measured in any given pixel. As the intensity will drop somewhat exponentially with distance from the central pixel, the contribution from outer pixels will be lower than one millionth of the central pixel. However, the dynamic range of the CCD camera is limited to 16 bit or 65536 digital counts. To circumvent this problem, the PSF is determined using a homogeneous monocrystalline silicon solar cell as an electroluminescence source and taking several images with a number of apertures of different size. Images taken for instance with a very large aperture allowed very small values of the PSF at large distances from the centre pixel to be determined. On the other hand, the smallest aperture, a $0.1 \mathrm{~mm}$ diameter pinhole, only allowed PSF values in the immediate vicinity of the centre pixel to be determined. We measure up to five different aperture areas ranging from 25 to $0.1 \mathrm{~mm}$ and scale according to a factor linear to the product of exposure time and aperture area. We fit the resulting experimental PSF with an exponential decay function which is then used to generate a two dimensional $1024 \times 1024$ pixel PSF image. The PSF image needs to be normalised to a total sum equal to unity to ensure that the total count rate of both convoluted and deconvoluted images are equal. The Richardson-Lucy and the Wiener deconvolution methods are the most common non-iterative deconvolution algorithms. ${ }^{30,31}$ For this study we use direct reverse filtering without regularisation as it is build-in ImageJ. ${ }^{32,33}$

We note that the PSF is specific for each silicon CCD chip and is dependent on its design but is found to show similar properties. ${ }^{33}$ An experimental PL imaging system at UNSW is used for the experiments in this work employing a one megapixel CCD camera (Finger Lakes Instrumentation PL4710) which sensor features $13 \times 13 \mu \mathrm{m}^{2}$ sized pixels. The electronic gain of the camera is measured to be close to one, meaning one photon absorption results in one digital count, thus utilising the weak luminescence from the unpassivated brick surface well, if absorbed. This is in good agreement with gain factors given in Hinken et al. ${ }^{34}$ The chip is backilluminated, deep-depleted and utilises a near infrared (NIR) anti reflection coating for enhanced silicon luminescence absorption. Precise thermoelectric cooling keeps a constant CCD operation temperature of $243 \pm 0.05 \mathrm{~K}$ for all measurements shown in this work. The corresponding quantum efficiency of the camera at $243 \mathrm{~K}$ is displayed in Figure 2. Laser light of $880 \mathrm{~nm}$ wavelength with a spectral width (FWHM) of $2.8 \mathrm{~nm}$ is used to excite the silicon brick with 1 sun equivalent power $\left(0.1 \mathrm{~W} / \mathrm{cm}^{2}\right)$. Beam shaping optics are employed to generate a uniform excitation, and filtering is applied at the homogenizer exit and in front of the camera lens as both described previously (see Ref. 34). We choose a lens with high optical power $(\mathrm{f}=1.4)$ and a relatively long focal length of $50 \mathrm{~mm}$ to limit lens artefacts. A dark black box surrounding the sample and the illumination and detection unit ensures no stray light is measured. The geometry of the experimental apparatus is equivalent to the one presented in Fig. 1 in Ref. 34.

We characterise the spectral sensitivity of the setup carefully by measuring all filter characteristics with a Perkin-Elmer photo spectrometer and verify the quantum efficiency data of the silicon CCD as supplied by manufacturer (see Figure 2). As the measurement of quantum efficiencies

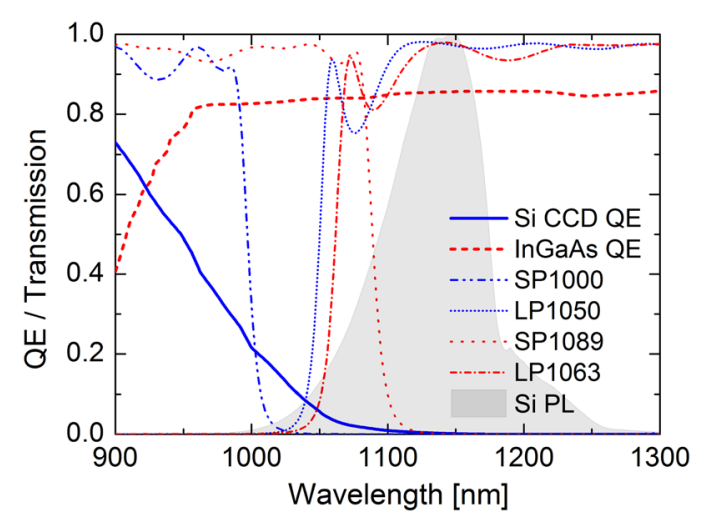

FIG. 2. Camera quantum efficiencies (QE) and filter transmission characteristics of the experimental apparatus used in this study. The luminescence emission spectrum of a silicon brick of $100 \mu$ s bulk lifetime normalised to its maximum intensity is given as guidance to the eye. 


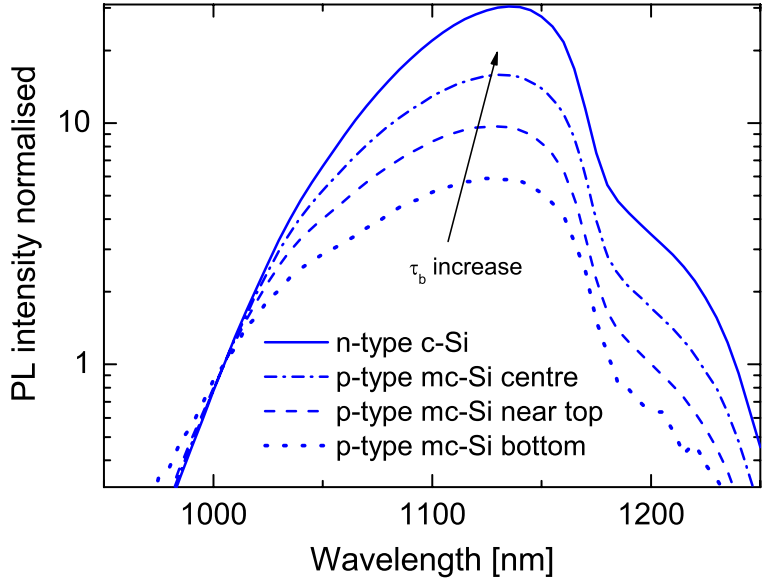

FIG. 3. Photoluminescence spectra measured on polished and unpassivated silicon brick side faces in areas of increasing bulk lifetimes. The illustrated spectra are normalised to their intensity at $1000 \mathrm{~nm}$ in arbitrary units and shown on a semi-logarithmic scale. Prominent defect luminescence peaks in the wavelength range $1150-1300 \mathrm{~nm}$ are not detected in any lifetime region.

below $1 \%$ is expected to be inaccurate, we extrapolate using Beer-Lambert law as specified in Ref. 1.

\section{InGaAs camera}

A cooled complementary metal oxide semiconductor (CMOS) indium gallium arsenide (InGaAs) camera is an alternative to employing a silicon CCD camera. Due to the lower band gap of InGaAs the spectral sensitivity extends from 950 to $1700 \mathrm{~nm}$ and thus covers the entire spectral range of band-to-band luminescence from crystalline silicon, the latter most importantly with negligible spectral variation (see Figure 2).

An InGaAs camera incorporates two potential advantages compared to silicon CCD cameras when applied to the PLIR method. First, the quantum efficiency is effectively uniformly high from 950 to $1300 \mathrm{~nm}$, which removes a source of uncertainty in the quantitative analysis, i.e., the knowledge of the exact spectral response of the sensor (see Figure 2). Second, the absorption coefficient of InGaAs is orders of magnitude higher across the entire spectral range of the silicon luminescence, which significantly reduces optical image distortions due to light spreading within the camera chip and increases the photocurrent generation in the detector. Using an InGaAs camera, the above optical artefacts can be expected to be negligible, which should allow using PL intensity ratios without the need for deconvolution.

InGaAs cameras have been used for dynamic infrared lifetime imaging ${ }^{35,36}$ and recently applied to photoluminescence imaging for the analysis of lifetime ${ }^{9}$ and defect polarisation. ${ }^{37}$ We use a Xenics camera, model XEVA1.7-640 with a $640 \times 512$ array of $20 \times 20 \mu \mathrm{m}^{2}$ pixels. The electronic gain in the employed high gain setting is specified by the manufacturer to be 14 analog to digital counts, thus significant larger than the silicon CCD camera, but was not measured in this study. We employ an $\mathrm{f}=1.4$ lens with $28 \mathrm{~mm}$ focal length with this camera type, which maximises the luminescence flux onto the pixel. This in consequence maximises the signal to noise ratio and further eliminates the need for image stitching. A detailed study of the noise statistics of the same InGaAs camera in comparison to a similar silicon CCD array is reported in Hinken et al. ${ }^{34}$ One important additional contribution to the noise is the fact that a CMOS camera, as the InGaAs camera is, features a signal amplifier for each pixel, thus causing the need for a calibration of each individual pixel gain factor. This is in fact one of the shortcomings in comparison to the CCD technology resulting in less efficient photon to electronic count transfer (higher gain), limited exposure times, and going along with higher costs.

\section{RESULTS AND DISCUSSION}

\section{Photoluminescence spectroscopy}

To verify any relevant impact of a spectral defect luminescence signal on the spectral photoluminescence intensity ratio imaging method, we measure non-spatially resolved photoluminescence spectra on various locations on both mono- and multicrystalline silicon bricks.

Figure 3 shows the resulting spectra taken on locations with increasing bulk lifetime, including one location each of in the impurity rich, so-called "red zones" from the top and bottom regions of a multicrystalline silicon brick. The latter areas were found to be most strongly affected by artefacts in our previous study. ${ }^{1}$

We find no evidence for significant defect luminescence in any of the spectra including spots measured in the red zone. A significant impact of defect luminescence on the

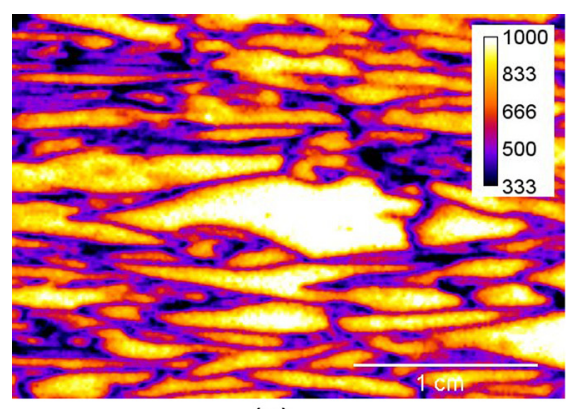

(a)

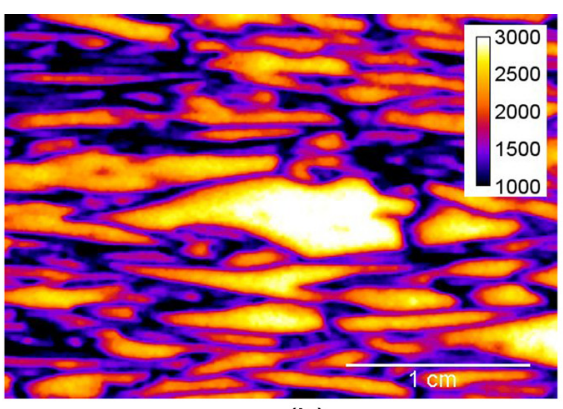

(b)

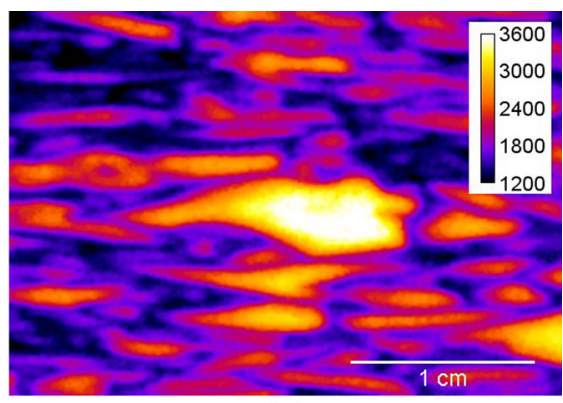

(c)

FIG. 4. Three qualitative PL images of the same area obtained with different luminescence light filtering shown in same relative scale: (a) short pass 1000 nm, (b) short pass $1025 \mathrm{~nm}$, (c) long pass $1050 \mathrm{~nm}$. The image contrast is blurred more noticeably with increasing filter wavelength using a silicon CCD camera. Given digital count rates correspond to an exposure time of $10 \mathrm{~s}$. 


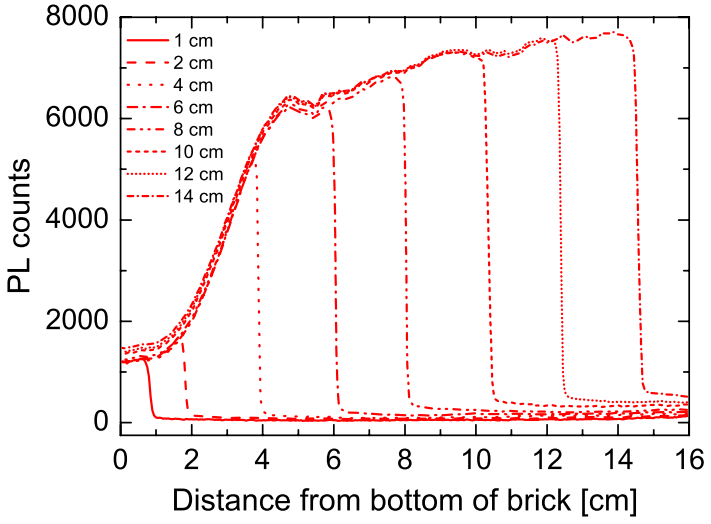

(a)

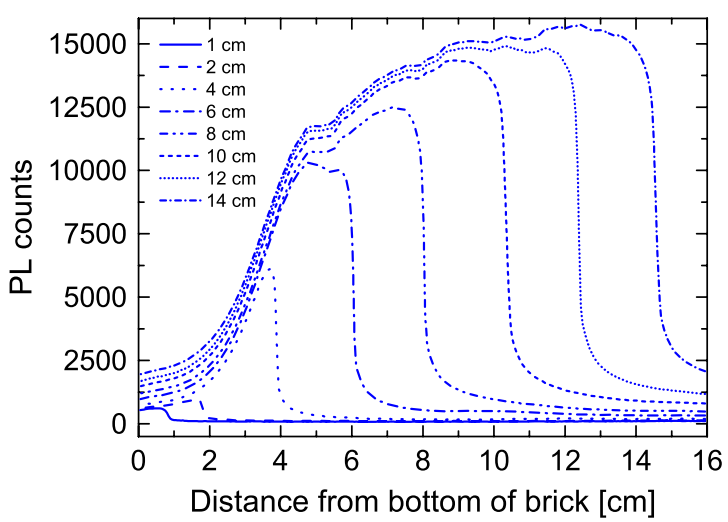

(b)

FIG. 5. Average cross-sectional luminescence intensity of the bottom section of a silicon brick side face as a function of distance to the bottom of the brick. Data is plotted for increasing areas of exposed silicon starting from the bottom of the brick, visualising the light spread for both short (a) and long pass (b) filtered images (SP1000, LP1050). The signal in the low lifetime bottom is increased due to light spreading from higher signal areas and particularly profound in the long pass images. Both graphs (a) and (b) reveal significant light spreading into the masked areas.

quantitative accuracy of the PLIR method can therefore be ruled out.

Room temperature D-bands are found to also not affect the method significantly when measuring with an InGaAs camera. A simple test comparing the intensity measured with and without a short pass filter with a cut-off wavelength of $1350 \mathrm{~nm}$ showed to not change the intensity ratios.

A full spectrum assessment by comparison with Eq. (1) allows calibration into local bulk lifetime. The detailed analysis of these spectra will be presented in a separate publication.

\section{Optically induced blur}

As discussed above, light spreading within the silicon CCD chip occurs and is expected to cause the image blurring to be more pronounced for longer wavelengths. Figure 4 shows three PL images taken on the same sample area of a silicon brick side face but with different spectral filters. It can clearly be seen that the image contrast is blurred most severely in the image taken with the $1050 \mathrm{~nm}$ long pass filter. The optical effect will be analysed and quantified in the following sections. The electrical diffusion as the driver for electrical blur alongside grain boundaries and dislocations accounting for some of the contrast change in Figure 4 will be discussed in a separate publication.

\section{Masking experiment}

We perform a simple masking experiment investigating the optical light spread from the high signal central to the low signal top and bottom parts of the brick. A series of images are taken for each filter starting with just the bottom centimetre of the brick being exposed. Subsequently, more and more of the brick side face is exposed the field of view of the camera. Figure 5 illustrates the resultant average crosssectional PL intensities shown as a function of distance to the bottom of the brick for increasing areas of exposed brick face.

We find considerable light spreading for both filters. The short pass filtered images reveal a significantly lower absolute spreading at both edges and into masked areas. Light spreading within the long pass filtered images can account for a dominant part of the signal in the bottom of the brick. As low lifetime areas dominated, central areas are affected as well, but to a significantly lesser degree as Figure 5(b) illustrates. We note that this experiment, in difference to the other experiments, uses a lens with a larger f-number of 2.8 and a focal length of $50 \mathrm{~mm}$ resulting in lower count rates, but should not affect the qualitative result.

\section{Quantifying light spreading}

We choose an experimental approach to quantify this light spreading effect within the optical detection unit employing a silicon CCD array and to measure its PSF as we describe in the theory section.

Figure 6 shows electroluminescence images of a monocrystalline silicon solar cell masked by a $0.1 \mu \mathrm{m}$ diameter pinhole, taken with a $1000 \mathrm{~nm}$ short pass filter and with a $1063 \mathrm{~nm}$ long pass filter, respectively (see Figure 2). As expected, we observe the image taken with a short pass filter shows limited blur, with preference to the $\mathrm{x} / \mathrm{y}$ neighbours, whereas the long passed light reveals a significant light spread across several pixels with a slower lateral reduction in intensity.

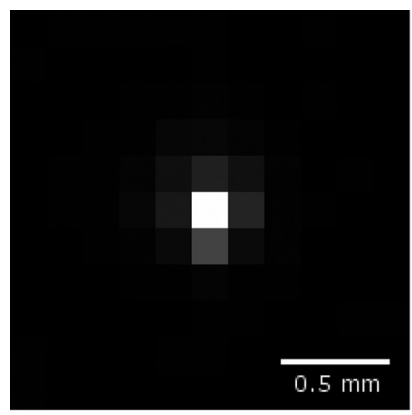

(a)

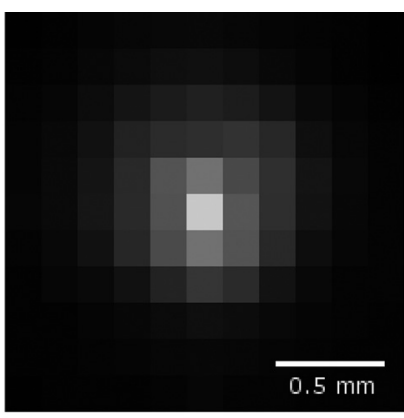

(b)
FIG. 6. PL images of luminescence light penetrating through a pinhole of $100 \mu \mathrm{m}$ in diameter placed in the centre of one pixel in the central part of the detecting CCD array. The luminescence light is filtered with a short pass $1000 \mathrm{~nm}$ in (a) and a long pass $1063 \mathrm{~nm}$ in (b) and shown in the same relative intensity scale. 


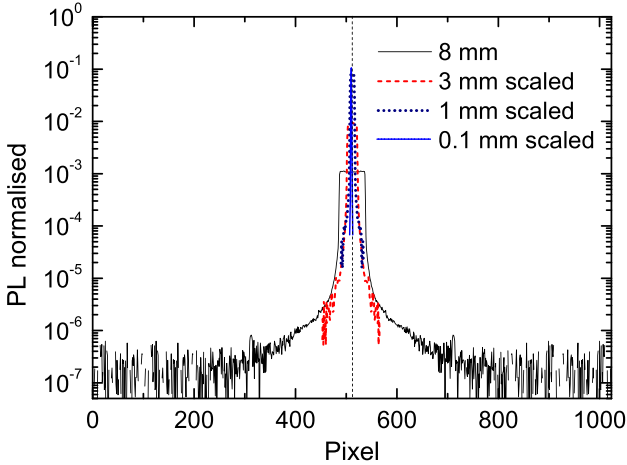

(a)

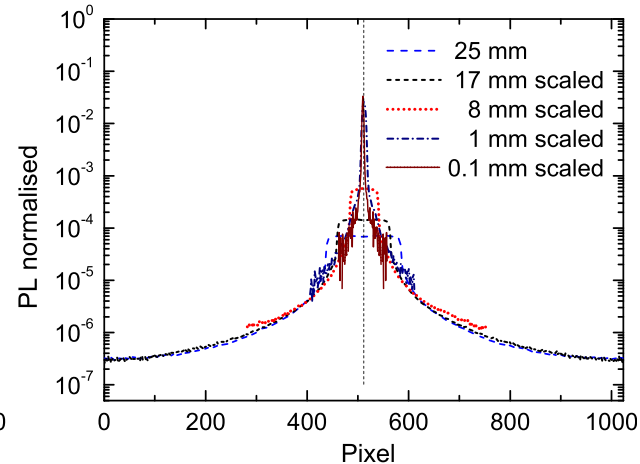

(b)
FIG. 7. Experimental point spread functions for short pass (a) and long pass (b) filtered PL images (SP1000, LP1063) using the described experimental apparatus. Up to five circular apertures are measured and scaled resulting in a PSF covering up to 7 orders of magnitude.
We apply the above procedure to measure the PSF for images taken with a $1000 \mathrm{~nm}$ short pass filter and with a $1063 \mathrm{~nm}$ long pass filter, respectively. Resulting cross sections through the PSF are shown in Figure 7 revealing a significantly stronger spread in the case of the images taken with the long pass filter.

We best fitted the PSF with an exponential decay function consisting of three terms

$$
y=y_{0}+A_{1} e^{-\frac{x}{t_{1}}}+A_{2} e^{-\frac{x}{t_{2}}}+A_{3} e^{-\frac{x}{t_{3}}} .
$$

While the initial decay across the first few pixel might be ruled by the electrical smear in the charge transfer process,$^{38}$ most of the decay is expected to be dominated by optical absorption following Lamberts law. The decay once not effected by electrical smear does not simply follow a single term exponential function, which would appear as a linear decay in Figure 8(a), since the absorption coefficient is wavelength dependent and the total decay curve is the convolution of each individual PSF for each wavelength with the experimental setup and the luminescence spectrum defining the spectral intensities.

We next use the obtained analytical functions (see Figure 8(a)) for the calculation of two dimensional PSF images. Figure 8 (b) shows the ratio of the long divided by the short pass filtered PSF fitting functions. The ratio reveals the light spreading of the long pass filtered image to be up to five times more severe peaking at 26 pixel or $4 \mathrm{~mm}$ apart from the central source of signal.

Both the original image and the PSF image are subsequently employed to calculate the deconvoluted image using the build-in functionality of the image processing program ImageJ. ${ }^{32}$

The impact of this deconvolution is demonstrated in Figure 9 for both filters. It is found that both filtered images strongly improve in image contrast and sharpness. The impact is particularly profound for the long pass filtered image, strongly reducing the dramatic blur of the "as measured" image. The long pass filtered image is expectedly found to be the limiting image in terms of defining the contrast and blur of the final bulk lifetime image.

The bulk lifetime image is then determined by division of the images measured either with short or long pass filter and subsequent conversion of the ratio into bulk lifetime using a theoretical transfer function, calculated as described in Mitchell et al. ${ }^{1}$ Figure 10 shows the bulk lifetime image of a multicrystalline silicon brick side face with a resolution of 1.3 megapixels with and without applying the deconvolution approach to the individual images. A direct comparison exposes the significant improvement of image contrast in the central part and dramatic quantitative changes in the top and bottom of the brick. The overestimation of lifetime in the highly impurity contaminated top and bottom regions of the brick is reduced.

Figure 11(a) shows the ratio of the two bulk lifetime images illustrated in Figure 10. The strongest impact of the deconvolution is thus in areas of high lateral luminescence gradient, e.g., near grain boundaries and dislocation clusters. Particularly strong variations are observed in the top/bottom regions of the brick. These low intensity regions form a relatively small area fraction of the total brick area. Combined with the large luminescence intensity contrast between these

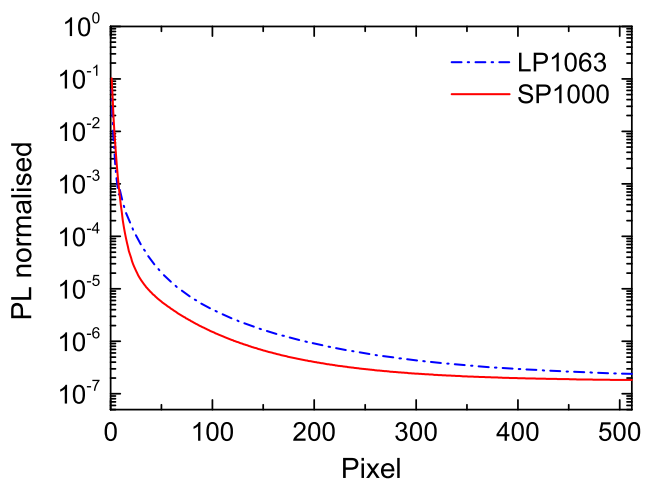

(a)

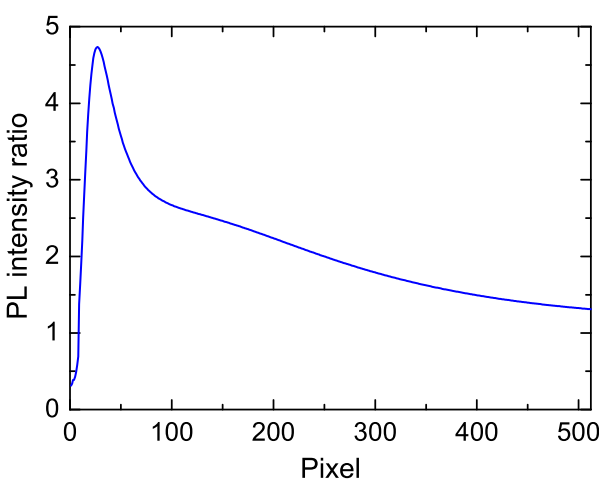

(b)
FIG. 8. (a) Fitting functions for both short and long pass filtered data from Figure 7 as used to determine the PSF image shown in semi-logarithmic scale. (b) The ratio of both PSF fitting functions indicates the characteristic of the relatively more severe light spreading of the long pass filtered image. Only one side of the bilaterally symmetrical curve is presented. The functions are specific to the experimental apparatus used in this study. 


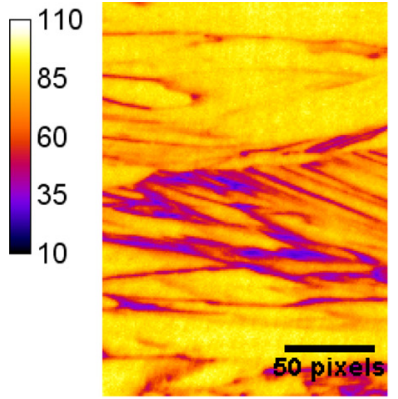

As measured

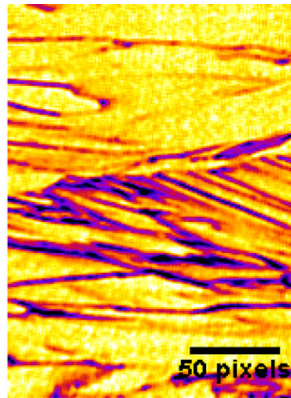

Deconvoluted

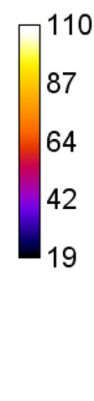

(a)

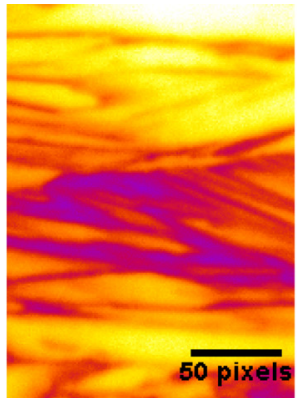

As measured

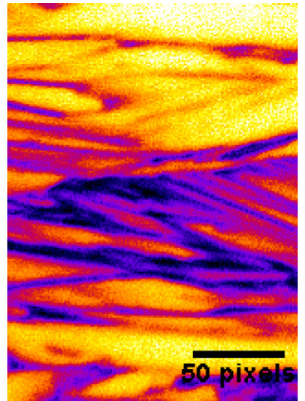

Deconvoluted

(b)

FIG. 9. Effect of image deconvolution is shown for images taken with both short pass SP1000 (a) and long pass LP1063 (b) filter revealing a pronounced improvement in image contrast. The noise level of the deconvoluted images is notably increased. The images are scaled for comparison. The signal is measured with up to 11000 counts in (a) and up to 35000 counts in (b).

low lifetime regions and the centre section of the brick, these areas are particularly affected by blurring inside the CCD. Cross sections through the bulk lifetime images from Figure 10 show bulk lifetimes below $1 \mu$ s in the bottom and top fraction, which is consistent with previous results. ${ }^{39}$ The long distance spreading of the high intensity luminescence from central areas of the brick has thus been corrected successfully leading to a better agreement with photoconductance measurements. ${ }^{14,15}$ Light spreading within the CCD can accordingly be unambiguously identified as the main cause for these artefacts.

The light spreading into the top and bottom can be effectively eliminated by masking the central parts of the brick during measurement as shown above. Masking trials allowed accurate measurement of the bulk lifetime of the top and bottom parts of the brick, demonstrating the fundamental ability of the method to detect lifetimes over a broad range from 0.01 to $10000 \mu$ s if employing a silicon CCD. We detect bulk lifetimes of below $0.01 \mu \mathrm{s}$ in the bottom of the brick. These

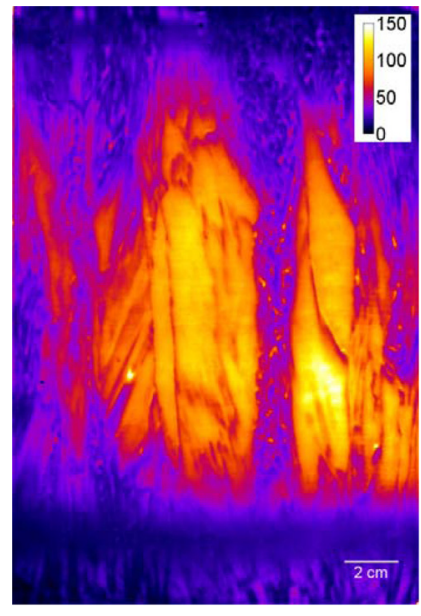

(a)

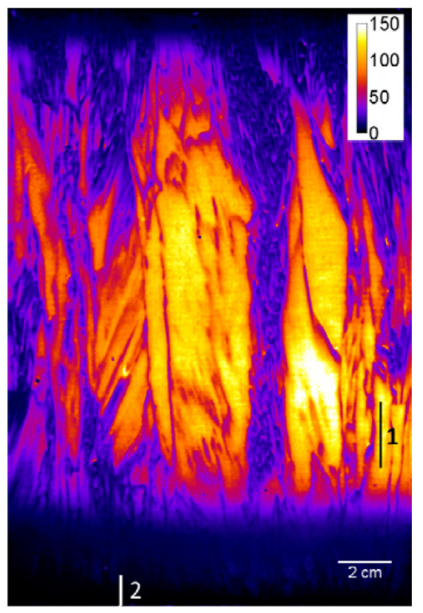

(b)
FIG. 10. Bulk lifetime image of a directionally solidified multicrystalline silicon brick side face without (a) and with (b) optical deconvolution of the individually filtered images. Both images taken within a total exposure of $120 \mathrm{~s}$ are shown on the same colour scale for direct comparison. The data is given in $\mu$ s with a resolution of 1.3 megapixels. In the deconvoluted image (b) two lines indicate the location of the line scan data taken for the noise analysis. values are confirmed similarly on a high impurity brick face originating from the edge of the ingot.

The comparison of the cross sectional values of Figure 11 show that the deconvolution improves the quantitative result overall and in the top and bottom dramatically, but cannot account for all of the light spreading into this low lifetime area if compared to masking data. It can be concluded that if exact bulk lifetime measurement of the bottom and top are needed, masking the central areas of the brick can give the most accurate results.

This study measured the PSF using a typical electroluminescence spectrum resulting in a first order correction since it is shown that the PSF image depends on the detection wavelength. As the emission spectrum itself depends on the local lifetime, a deconvolution that uses a PSF determined using a different source, will lead to some level of inaccuracies. A detailed investigation of these dependencies is beyond the scope of this paper.

\section{InGaAs detected bulk lifetime}

We use an InGaAs camera to measure the same silicon brick side face as shown in Figure 10. The PLIR method is applied to this camera type for the first time making it necessary to adapt the modelling towards the spectral sensitivity of the camera sensor as well as different lens and filters (see Figure 2).

Initial experiments indicated the necessity to change the set of filters for this camera type since the weak luminescence below $1000 \mathrm{~nm}$ is not sufficient to gain a reasonable signal to noise ratio (SNR). Therefore, we choose a longer cut-off wavelength for the short pass filter (here short pass at 1089 nm, see Figure 2). The InGaAs cameras available today require this broad luminescence band for sufficient photon flux onto the sensor pixels. The PLIR transfer function calculated for the InGaAs based detection system is presented in Figure 12 in comparison to the one calculated for the silicon CCD setup. The PL intensity ratio measured with the InGaAs camera is higher, but the gradient, as the most important feature for reliable transfer of PLIR into bulk lifetime, is significantly lower for most of the lifetime range relevant for PV applications. This is a direct result of the needed short pass 


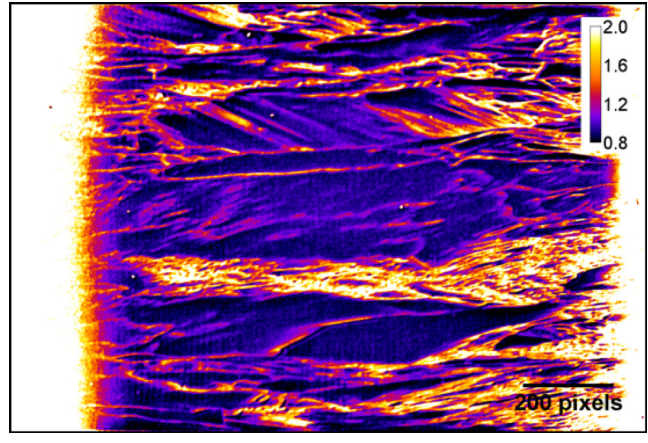

(a)

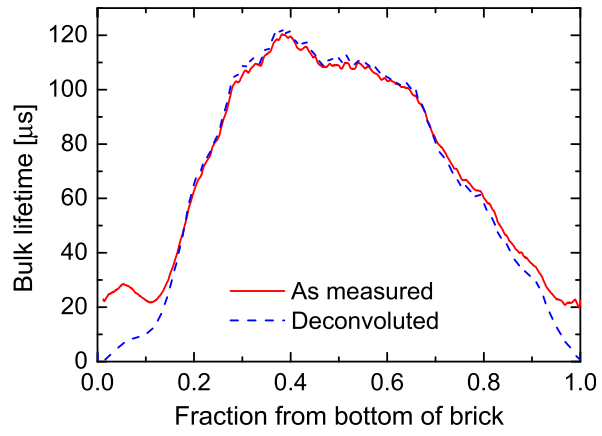

(b) filter selection and means that the accuracy for bulk lifetime measurements below around $100 \mu$ s is limited, whereas the silicon CCD still shows a solid gradient in the transfer function down to below $0.1 \mu \mathrm{s}$. Future developments in camera technology might allow using a short pass filter with a shorter cut off wavelength which would lead to a more accurate measurement for lifetimes below $100 \mu \mathrm{s}$.

The InGaAs camera is cooled and the pixel amplifiers are calibrated at $255 \mathrm{~K}$. We take both images either filtered with long or short pass with $2.5 \mathrm{~s}$ exposure time, correct the flat field, and divide to obtain the intensity ratio. After converting the ratio into bulk lifetime using the modelled transfer function shown in Figure 12 we obtain a bulk lifetime image as pictured in Figure 13.

The resulting bulk lifetime image reveals a good qualitative agreement with the silicon CCD detected image obtained from deconvoluted images, illustrated in Figure 10, especially in the central part of the brick. The InGaAs camera is not affected by optical blur, which is due to the high absorption throughout the silicon luminescence spectrum. Therefore, the image shows clear edges of grain boundaries, and smaller structures are not blurred out as in the silicon detected image.

The resulting bulk lifetime image is less highly resolved (0.2 instead of 1.3 megapixels) and reveals more visible noise in comparison to the silicon CCD image; thus, it appears less brilliant. We are not able to analyse the top and bottom parts of the brick analysed quantitatively due to the low SNR (see Table I). However, even if the signal would be sufficient, the flat transfer function in areas of low lifetime would limit the evaluation.

\section{Comparison of both camera types}

\section{Bulk lifetime topography}

Bulk lifetime data taken from a horizontal line scan located centrally on the brick side face, as indicated in Figure 13, is directly comparing the bulk lifetime contrast of the silicon CCD detected and deconvoluted image (see Figure 10) with the InGaAs detected image (see Figure 13) and is illustrated in Figure 14. The line scan reveals that overall bulk lifetime contrast agrees well, with smaller feature contrast, but also more noise shown in the InGaAs image. The silicon contrast is still comparably blurred especially in narrow features as between 0 and 0.3 and at 0.9 relative positions. This indicates that the deconvolution can only partially reverse the light spreading in the CCD. We can also observe that the InGaAs image is notably distorted due to the use of a $28 \mathrm{~mm}$ focal length lens resulting in a slight mismatch of the features. A larger absolute mismatch towards the side of the image gives indication of a possible impact of the edge of the CCD on the PSF and a resulting poor effectiveness of a single deconvolution, which does not account for its potential spatial variation. Moreover, the impact of light spreading on the flat field correction, especially of the long pass filtered image, cannot be ruled out fully with the application of a deconvolution algorithm.

Comparing data in vertical growth direction reveal lower lifetime gradients towards the top and bottom of the brick in the InGaAs detected image as the cross sectional analyses reveals. There are two possible reasons for this to occur. First, the deconvolution shows limitations to fully account for light spreading from the central high lifetime to the top and bottom low lifetime since the difference in signal is almost 2 orders of magnitudes higher centrally. Second, the flattening gradient of the InGaAs PLIR transfer function in conjunction with a decreasing signal to noise leads to uncertainties in the intermediate and low bulk lifetime areas.

The combined evaluation of both silicon CCD and InGaAs detected bulk lifetime images along with the single images indicate a somewhat sensitivity of the silicon detected images towards the surface properties as Figure 15(a) visualises. The

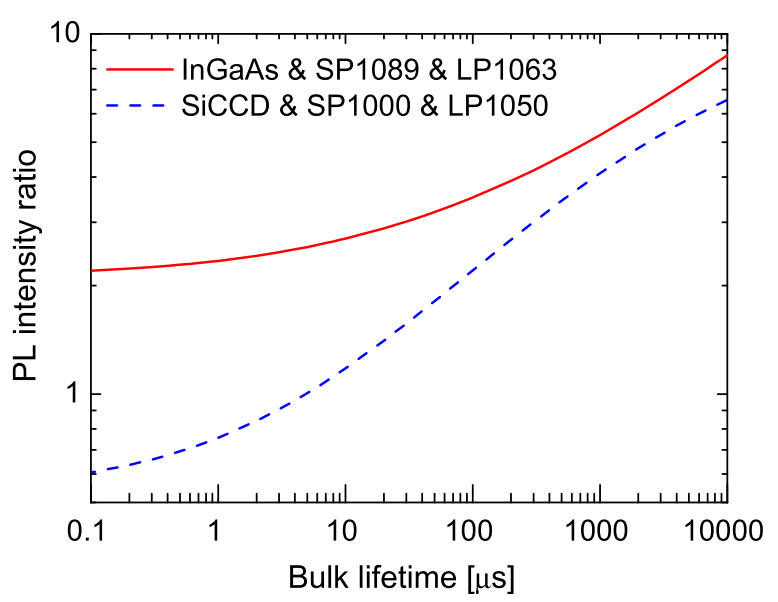

FIG. 12. Photoluminescence intensity ratio transfer functions calculated for two different detection systems consisting of a unique detector, filters, and lens. Both systems show similar gradients for high lifetimes above $500 \mu \mathrm{s}$, but the InGaAs camera with its selected filter combination reveals significantly lower gradients for lower bulk lifetime values, resulting in reduced sensitivity to bulk lifetime variations. 


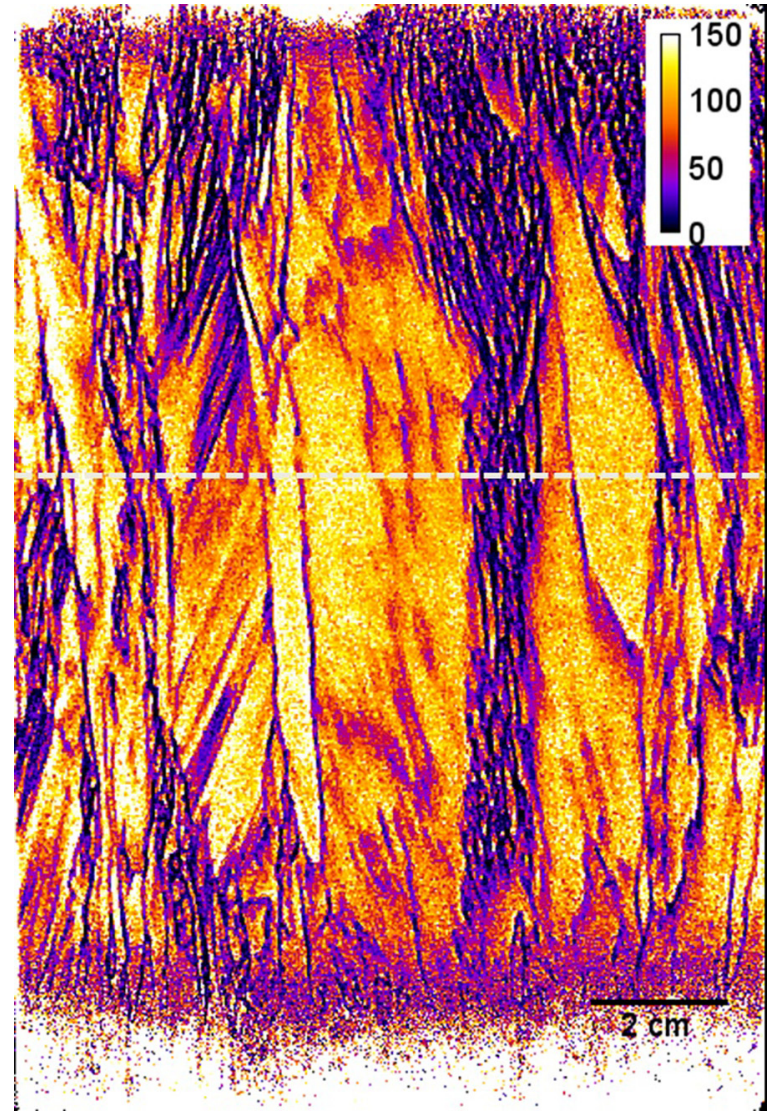

FIG. 13. Bulk lifetime image of the same brick face as shown in Figure 10 obtained from the PLIR method utilising an InGaAs camera. The image is taken with a total exposure time of just $5 \mathrm{~s}$ and shows a resolution of 0.2 megapixels. The colour scale gives the absolute bulk lifetime in $\mu$ s. Good qualitative and quantitative agreement with Figure 10 is observed with less blur but higher noise affecting the image. The bottom and top of the brick, here mostly appearing in white, could not be evaluated quantitatively due to a very low signal to noise ratio. The dashed line indicates the line scan data shown in Figure 14.

image illustrates a normalised silicon CCD detected images filtered with a $1000 \mathrm{~nm}$ short pass. The circular structures of the mechanical polishing step prior to the measurement appear if we scale the image as labelled, thus indicating that mechanically induced surface properties reflect in the detected luminescence signal. Figure 15(b) shows the equivalent InGaAs detected images filtered with a $1089 \mathrm{~nm}$ short pass. This image

TABLE I. Experimental data of the signal to noise ratios (SNR) for both the silicon $\mathrm{CCD}$ and the InGaAs camera for the respective line scans as indicated in Figure 10. In the case of the silicon CCD data is also given after deconvolution.

\begin{tabular}{lcccc}
\hline \hline & & Linescan 1 & Linescan 2 \\
\hline \multirow{3}{*}{ Silicon } & & $\mathrm{t}_{\mathrm{exp}}[\mathrm{s}]$ & SNR & SNR \\
& SP raw & 60 & 37 & 26 \\
& SP deconvoluted & 60 & 7.5 & 2.2 \\
& LP raw & 60 & 60 & 43 \\
& LP deconvoluted & 60 & 24 & 3.5 \\
InGaAs & SP raw & 2.5 & 21 & 1.4 \\
& LP raw & 2.5 & 28 & 5.6 \\
\hline \hline
\end{tabular}

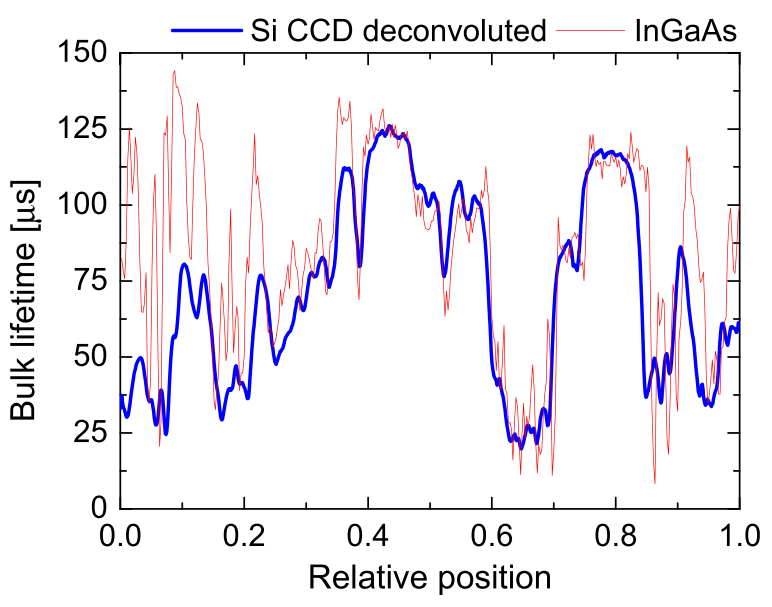

FIG. 14. Cross sectional comparison of InGaAs and Si CCD detected bulk lifetimes. The line scans are taken horizontally from the centre of the brick face as indicated in Figure 13. Qualitative agreement is observed with more contrast of small features visible in the InGaAs data. The contrast of the deconvoluted $\mathrm{Si}$ detected images still notably blurred in comparison here. Slight misalignment in the data is due to optical distortion in the InGaAs image which is taken with lens of shorter focal length.

does not reveal any surface polish structures drawing a more uniform signal contrast.

The inhomogeneity of the silicon detected short pass image is reflected in the final bulk lifetime image (see Figure 10), hence exhibiting features which are not represented in the InGaAs images and consequently lead to some of the mismatch of in the cross sectional data of Figure 14 in the area fraction $0-0.3$.

To estimate the average depth the luminescence signal origins from for both short pass filtered images we calculate the luminescence intensity as a function of depth as detected by the experimental apparatus used in this work (see Figure 16). To generate comparably data with Figure 15, we assume short pass filtering (SP1000/SP1089), no dead layer on the surface, an excitation at $880 \mathrm{~nm}$, a p-type doping of $1 \times 10^{16} \mathrm{~cm}^{-3}$, a sample temperature of $300 \mathrm{~K}$, and detector and filter characteristics as introduced in Figure 2.

We learn that the weighted average signal depth of the image shown in Figure 15(a) is merely above $100 \mu \mathrm{m}(117 \mu \mathrm{m}$ at $100 \mu$ s bulk lifetime), which results from the fact that we employ a shorter cut off wavelength and a silicon detector with a rapidly decreasing quantum efficiency towards longer wavelengths. Since the luminescence signal below $1000 \mathrm{~nm}$ does not change much with bulk lifetime, the weighted average signal depth is only weakly dependent on the bulk lifetime and the standard variation of the signal depth for this setup remains moderate with $39 \mu \mathrm{m}$ at $100 \mu$ s bulk lifetime.

In difference, the InGaAs detected image of Figure 15(b) features a significantly larger weighted average signal depth of mostly substantially more than $200 \mu \mathrm{m}(283 \mu \mathrm{m}$ at $100 \mu$ s bulk lifetime) accompanied by a larger dependency on the local bulk lifetime and significantly enhanced standard variation of $134 \mu \mathrm{m}$ at $100 \mu$ s bulk lifetime.

This analysis allows us to understand the observed sensitivity of the luminescence image to surface defects if we employ a $1000 \mathrm{~nm}$ short pass filter. That is, since we effectively only utilise luminescence from a $100 \mu \mathrm{m}$ thick 


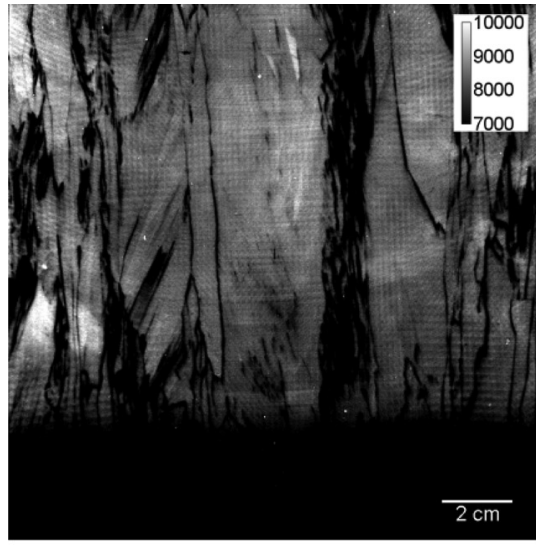

(a)

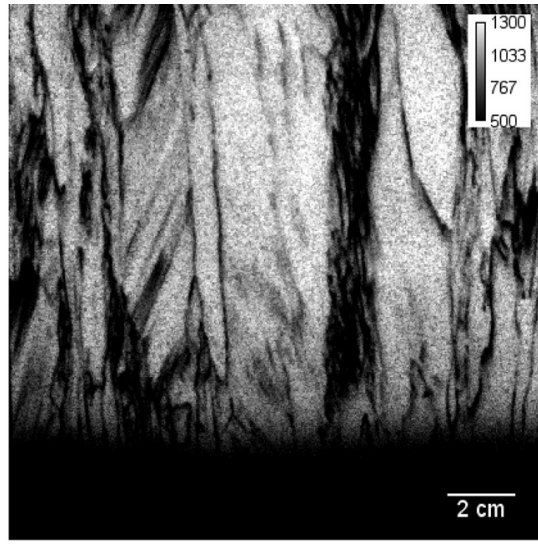

(b) surface layer. The latter thickness is mostly independent from the actual bulk lifetime, which consequently means that larger bulk lifetimes as we find in $\mathrm{Cz}$ grown ingots do not remove this surface sensitivity significantly, which can only be overcome by a uniform and non-destructive polish, careful clean of the surface, or a longer filter cutoff wavelength.

Furthermore, an estimation of the depth of surface damage on cast silicon bricks remains difficult but seems plausible to be up to $20-30 \mu \mathrm{m}$ in depth. The enhanced luminescence signal of some areas, as visible in Figure 15(a), may possibly be explained by a micro roughness (texture, micro scratches) due to an imperfect polish and therefore enhanced local emission coefficient, which is often induced by the surface geometry. A reduction in luminescence signal could be explained by an electronically dead layer as introduced during slicing process, which is possibly not fully or homogenously removed by the polish step.

\section{Quantitative bulk lifetime-Summary}

The photoluminescence intensity ratio imaging method allows obtaining quantitative bulk lifetime by analysing spectral changes in intensity.

The remaining limitations, in the case of the silicon camera, are mainly caused by the fact that silicon luminescence is measured with a silicon detector. The detector quantum efficiency quickly drops with increasing photon wavelength across the emitted spectrum. As a result only a fraction of the band-to-band luminescence is absorbed, and a precise knowledge of the quantum efficiency is needed to model the spectral intensity ratio. Any gradient in spectral sensitivity is a possible source of uncertainty since the transfer function is defined by the intensity ratio in different spectral intervals, which is directly dependent on variations in the quantum efficiency of the CCD array.

Precise measurement of the CCD quantum efficiency up to wavelengths of $1150 \mathrm{~nm}$ is a challenging task. The impact of the uncertainty in the spectral response data on the PLIR is estimated to be $\pm 5 \%$ on the intensity ratio, as further experiments showed. This is a revision to the value given by Mitchell et al. ${ }^{1}$ and leads to an uncertainty in the absolute bulk lifetime of up to $\pm 25 \%$ just caused by the CCD, not yet accounting for other sources of uncertainties (see Ref. 1). However, such uncertainties can be mitigated to a large extent by adapting the transfer function and referencing the result to a golden standard which can be calibrated with, e.g., PLIR using the InGaAs camera, microwave detected photoconductance (MPD), quasi steady state (QSSPC) or transient photoconductance (PCD), and surface photovoltage measurements (SPV).

Employing an InGaAs detector instead is estimated to lead to a more accurate quantitative result for three reasons. First, the camera chip does not show optical light spreading; thus, the images and the flat fields are not blurred as is the case with the silicon CCD images. Second, the spectral dependence of the InGaAs quantum efficiency in the wavelength range of the silicon luminescence emission is weak, thereby effectively eliminating the detector quantum efficiency as a source of

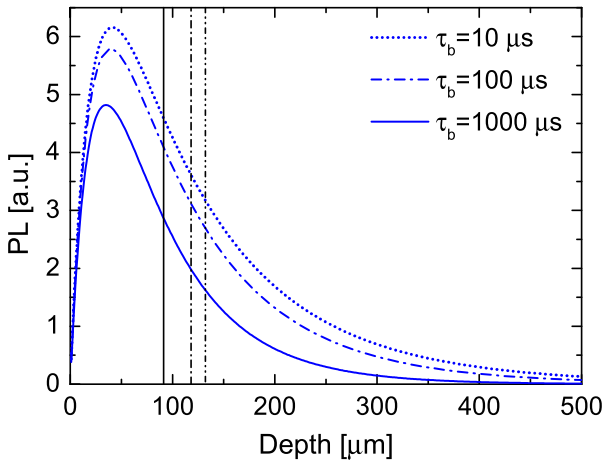

(a)

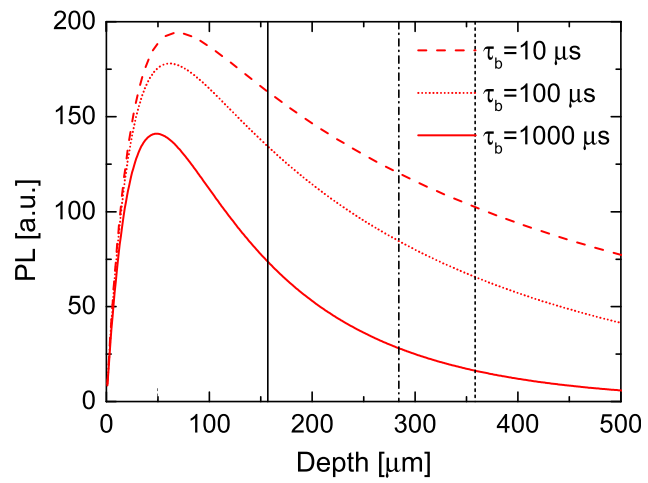

(b)
FIG. 16. Calculated origin of luminescence intensity as a function of depth and various bulk lifetimes corresponding to Figure 15. Illustrated profiles are (a) silicon CCD detected and short pass (SP1000) filtered and (b) InGaAs detected and short pass (SP1089 nm) filtered. The weighted mean signals depths are indicated with vertical lines for each bulk lifetime. The calculated total InGaAs photocurrent (filter and lens as noted) is 73 times higher at $100 \mu$ s corresponding bulk lifetime if assuming a lens with the same focal length. 
uncertainty. Finally, a short pass filter with a longer filter cutoff is used in the InGaAs setup, which reduces the impact of the surface.

The remaining shortcomings are, first, the low signal to noise ratios of this camera type limiting the quantitative data analysis to central high lifetime regions $\left(\tau_{\mathrm{b}}>25 \mu \mathrm{s}\right)$ as discussed in the following, and, second, the transfer functions starts flattening below $1 \mathrm{~ms}$. We estimate the total quantitative accuracy of the InGaAs detected bulk lifetime images to be within $\pm 15 \%$ relative for bulk lifetimes above $100 \mu$ s. In consequence, the silicon CCD remains more accurate for lifetimes below $100 \mu$ s with the discussed filter combination. However, as future more sensitive camera technologies based on direct semiconductors like are expected, a short pass filter with a cutoff at $1025 \mathrm{~nm}$ or band pass filter $1000-1025 \mathrm{~nm}$ could allow accurate bulk lifetime to be detected down below $1 \mu \mathrm{s}$.

\section{Noise analysis}

An experimental analysis of the camera noise is presented comparing the signal to noise ratios for both camera types for various conditions. There are four dominant sources of noise in photoluminescence imaging: First, the signal noise, often also referred as shot noise, is a result of the luminescence emission following the Poisson distribution in time, second the thermal generation of free carriers in the sensor, also called dark (shot) noise, further the read-out noise due to electronic amplification and conversion of the signal in to digital unit and finally the temperature fluctuations due to imperfect temperature stabilisation.

Both camera types have different values for each of these noise fluxes as discussed in detail for similar cameras in Hinken et al. $^{34}$ Even with a lower InGaAs chip temperature of $\mathrm{T}=255 \mathrm{~K}$, as chosen in this study, the dark and the temperature noise dominate the overall noise of the InGaAs camera measurements. As a result the signal to noise ratio does not improve for exposure times above $2.5 \mathrm{~s}$ and a calibration of the individual gain factors could not be reliably performed. The silicon CCD however is cooled precisely to $243 \mathrm{~K}$ allowing exposure times of up to $120 \mathrm{~s}$ causing the noise to be dominated by the photon (shot) noise. The silicon CCD SNR is therefore approximately proportional to the square root of the total dark corrected signal, which is linearly proportional to the exposure time.

Hinken et al. state the dark noise flux of the InGaAs chip to be up to 7 orders of magnitude higher than the silicon CCD dark noise. In the context of signal to noise, that higher dark noise negates to a large extent the benefit of an enhanced luminescence signal due to the better spectral response. This fact is critical especially in the application to low luminescence emitters like low lifetime silicon bricks.

We evaluate two line scans to access experimental measures of the noise apparent in the final camera count rates in terms of their apparent noise fluctuations for both camera types. The location of the line scans are indicated in Figure 10(b). Line scan 1 represents a high lifetime grain with a bulk lifetime of around $100 \mu$ s and line scan 2 is located in the very bottom of the brick, thus representing lifetimes below $0.5 \mu$ s. We take the data from the raw images without any prior noise filtering or smoothing. The influence of background lifetime variation throughout the scan is removed via the division of a linear or polynomial fit of the data. The resulting flat signal is then normalised to the mean value for comparison, and the standard deviation is obtained.

Figure 17 shows the data obtained and gives a clear picture of the apparent noise. In the case of the silicon CDD camera the noise levels are fairly low for both regions. Even the bottom and top of the brick can be measured with a SNR of above 25 (see Table I).

However, the deconvolution algorithm induces a significantly higher noise level by spreading localised noise throughout the image. This in principle can be avoided by employing regularisation, ${ }^{33}$ but has not been implemented in this work. The InGaAs detected images show reasonable low noise levels in the high lifetime area (SNR > 20), but the absolute counts in the bottom area especially for the short pass filtered images are too low to be differentiated from noise as a SNR of just 1.4 is found.

The data reveals the images measured with a short pass filter to show lower SNRs, which is mainly due to the lower absolute luminescence intensity transmitted through the filter. A longer cut off wavelength can be chosen, which is, however, limited since it reduces the gradient of the transfer function.

The bottom and top parts of the silicon brick could not be evaluated quantitatively with the InGaAs camera, since the signal level and the resulting SNR are too low. An increased photon flux by using higher power optics, and a more stable and lower detector temperature could allow sufficient count rates to be achieved in this area. Both these would also allow longer exposure times and higher SNR in the central area of the brick, thus enabling similar or exceeding ratios as to the silicon CCD based measurements.

\section{CONCLUSIONS}

A better understanding of the photoluminescence spectral intensity ratio method is achieved. Improvements of the method are suggested and demonstrated experimentally, and the applications of the technique are extended.

We confirm that defect luminescence is not responsible for previously reported experimental artefacts in low lifetime regions of silicon bricks. However, we find that light spreading in the silicon CCD heavily affects the intensity ratio analysis, resulting in image blurring particularly in the image taken with a long pass filter. We propose an image deconvolution method and demonstrate to reduce the impact of the light spreading substantially, thus allowing a more accurate qualitative and quantitative analysis. Short pass filtering at $1000 \mathrm{~nm}$ remains a limiting element since the image contrast is shown to be somewhat dependent on surface properties after mechanical polish.

Experiments and modelling using an InGaAs detector chip reveal it to be an attractive alternative with a superior quantitative accuracy for bulk lifetimes of currently above $100 \mu$ s and insignificant image blur. However, a noise analysis exposes limitations in terms of a quantitative analysis in low lifetime areas, which could be improved by reducing the detector temperature, for example. Future improvement of this 

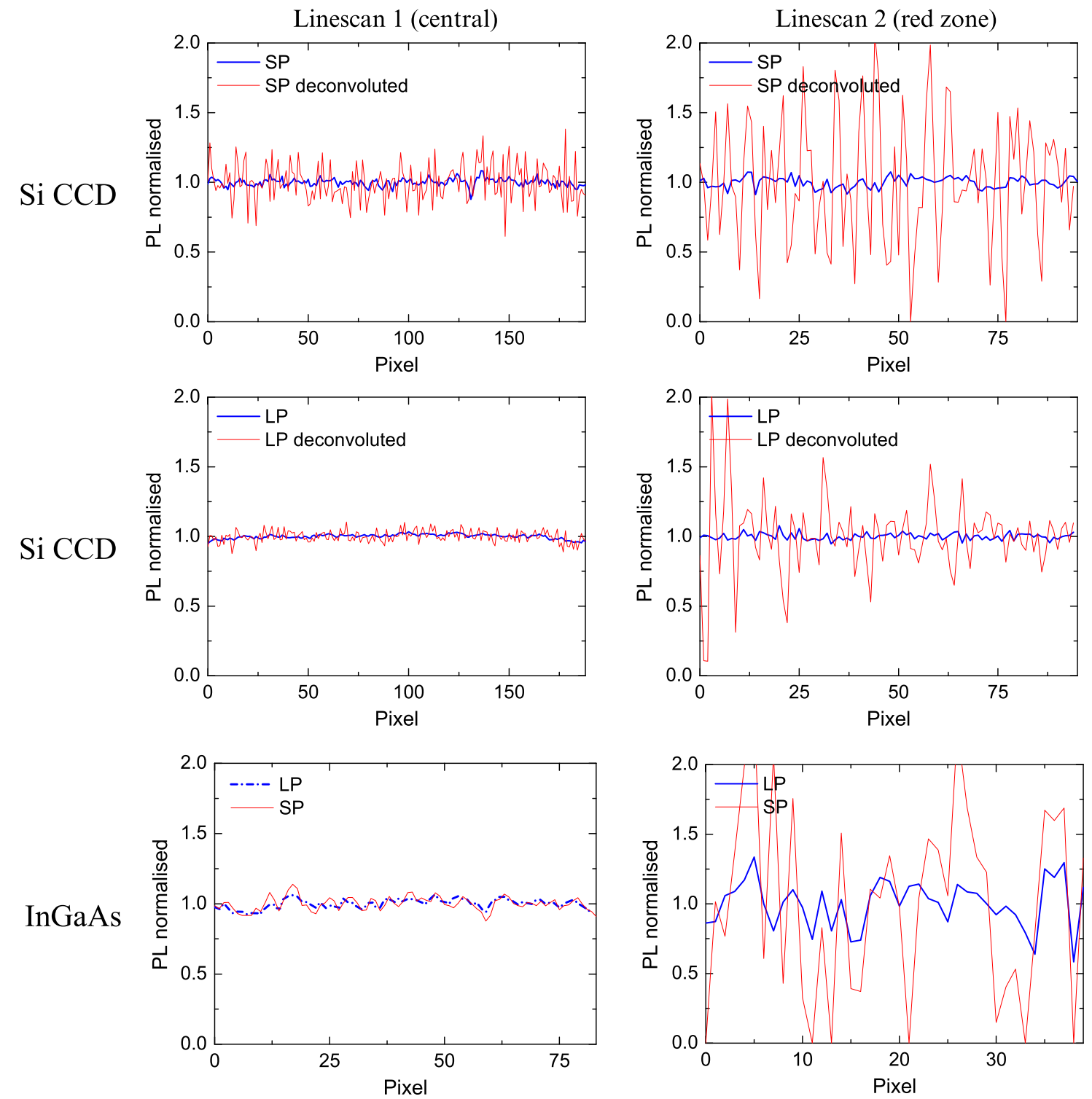

FIG. 17. Fluctuations of the digital count rates for both the silicon CCD and the InGaAs camera along two line scans. Data is given for both short and long pass image along line scan 1, representing a high lifetime region and line scan 2 taken in the bottom of the brick (red zone). The exact location of both line scans is indicated in Figure 10. All data is normalised to the mean and influence of lifetime signal is removed. The InGaAs camera shows a lower pixel resolution for the same line. Also noise levels after deconvolution are evaluated.

or related camera technology (e.g., GaInAsP) could place as an attractive alternative most likely with reduced measurement times towards the silicon CCD for comparable SNRs.

The comparison of both silicon CCD and InGaAs detected bulk lifetime images among each other and in addition with other measurements techniques (e.g., QSSPC) indicate that the deconvolution cannot reverse all of the effects of light spreading in the CCD array. Limitations remain in, first, resolving small features true bulk lifetimes, second, resolving the large gradients in bulk lifetime towards the bottom and top of the brick, and finally resolving the impact of light spreading on the flat field correction. Future studies should investigate the spectral, wavelength, and spatial dependence of the PSFs, the latter especially towards the edges of the CCD. Furthermore, advanced deconvolution algorithms, including the application of regularisation, need to be investigated.

Nonetheless, we believe that the improved and better understood method will allow the research and industry to more accurately image silicon bricks bulk lifetimes after solidification and slicing into bricks prior to wafer sawing. As multicrystalline and seeded cast silicon bricks play a major role in the cost reduction strategy of the industry and represent a significant market share. Bulk lifetime images can provide a fast and accurate early stage feedback of the crystallisation process and may allow prediction of device properties.

We note that the method is particularly well suited for the measurement of high lifetime Czochralski $(\mathrm{Cz})$ grown monocrystalline ingots. The effect of light spreading is low, the surface influence often minmal and the gradient of the transfer function is high. These conditions lead to accurate measurements.

\section{ACKNOWLEDGMENTS}

The authors would like to thank Jørgen Nyhus of REC Wafer for supply of silicon brick and Mattias Juhl for fruitful 
discussions. The work was supported by the Australian Research Council through the Photovoltaics Centre of Excellence.

${ }^{1}$ B. Mitchell, T. Trupke, J. W. Weber, and J. Nyhus, J. Appl. Phys. 109, 083111 (2011).

${ }^{2}$ T. Trupke, R. A. Bardos, M. C. Schubert, and W. Warta, Appl. Phys. Lett. 89, 044107 (2006).

${ }^{3}$ P. Wuerfel, T. Trupke, T. Puzzer, E. Schäffer, and W. Warta, J. Appl. Phys. 101, 123110 (2007).

${ }^{4}$ J. A. Giesecke, M. Kasemann, and W. Warta, J. Appl. Phys. 106, 014907 (2009).

${ }^{5}$ J. A. Giesecke, M. Kasemann, M. C. Schubert, P. Wuerfel, and W. Warta, Prog. Photovoltaics: Res. Appl. 18, 10-19 (2010).

${ }^{6}$ J. Haunschild, M. Glatthaar, M. Demant, J. Nievendick, M. Motzko, S. Rein, and E. R. Weber, Sol. Energy Mater. Sol. Cells 94, 2007 (2010).

${ }^{7}$ T. Trupke, J. Nyhus, and J. Haunschild, Phys. Status Solidi (RRL) 5, 131137 (2011).

${ }^{8}$ D. Kiliani, G. Micard, B. Steuer, B. Raabe, A. Herguth, and G. Hahn, J. Appl. Phys. 110, 054508 (2011).

${ }^{9}$ S. Herlufsen, K. Ramspeck, D. Hinken, A. Schmidt, J. Müller, K. Bothe, J. Schmidt, and R. Brendel, Phys. Status Solidi (RRL) 5, 25-27 (2011).

${ }^{10}$ P. Gundel, F. D. Heinz, M. C. Schubert, J. A. Giesecke, and W. Warta, J. Appl. Phys. 108, 033705 (2010).

${ }^{11}$ T. Trupke and R. A. Bardos, in 31st IEEE Photovoltaic Specialist Conference, Florida, USA, 2005, pp. 903-906.

${ }^{12}$ T. Trupke, R. A. Bardos, and M. D. Abbott, Appl. Phys. Lett. 87, 184102 (2005).

${ }^{13}$ S. Bowden and A. Sinton, J. Appl. Phys. 102, 124501 (2007).

${ }^{14}$ R. A. Sinton, T. Mankad, S. Bowden, and N. Enjalbert, in Proceedings of the 19th European Photovoltaic Solar Energy Conference, Paris, France, 2004, pp. 520-523.

${ }^{15}$ J. S. Swirhun, R. A. Sinton, M. K. Forsyth, and T. Mankad, Prog. Photovoltaics: Res. Appl. 19,313-319 (2011).

${ }^{16}$ M. A. Green, Appl. Phys. Lett. 99, 131112 (2011).

${ }^{17}$ M. A. Green, Sol. Energy Mater. Sol. Cells 92, 1305-1310 (2008).

${ }^{18}$ S. M. Sze and J. C. Irvin, Solid-State Electron. 11, 599-602 (1968).

${ }^{19}$ M. C. Schubert, J. Schön, P. Gundel, H. Habenicht, W. Kwapil, and W. Warta, J. Electron. Mater. 39, 787-793 (2010).

${ }^{20}$ P. Gundel, M. C. Schubert, W. Kwapil, J. Schön, M. Reiche, H. Savin, M. Yli-koski, J. A. Sans, M.-C. Gema, W. Seifert, W. Warta, and E. R. Weber, Phys. Status Solidi (RRL) 3, 230-232 (2009).
${ }^{21}$ R. P. Schmid, D. Mankovics, T. Arguirov, M. Ratzke, T. Mchedlidze, and M. Kittler, Phys. Status Solidi (a) 208, 888-892 (2011).

${ }^{22}$ T. Trupke, R. A. Bardos, M. D. Abbott, P. Würfel, E. Pink, Y. Augarten, F. W. Chen, K. Fisher, J. E. Cotter, M. Kasemann, M. Rüdiger, S. Kontermann, M. C. Schubert, M. The, S. W. Glunz, W. Warta, D. Macdonald, J. Tan, A. Cuevas, J. Bauer, R. Gupta, O. Breitenstein, T. Buonassisi, G. Tarnowski, A. Lorenz, H. P. Hartmann, D. H. Neuhaus, and J. M. Fernandez, in Proceeding of the 22nd European Photovoltaic Conference, Milan, Italy, 2007.

${ }^{23}$ M. C. Schubert, W. Kwapil, J. Schön, H. Habenicht, M. Kasemann, P. Gundel, M. Blazek, and W. Warta, Sol. Energy Mater. Sol. Cells 94, 1451-1456 (2010).

${ }^{24}$ T. Mchedlidze, W. Seifert, M. Kittler, A. T. Blumenau, B. Birkmann, T. Mono, and M. Müller, J. Appl. Phys. 111, 073504 (2012).

${ }^{25}$ M. Inoue, H. Sugimoto, M. Tajima, Y. Ohshita, and A. Ogura, J. Mater. Sci.: Mater. Electron. 19, 132-134 (2008).

${ }^{26}$ H. Sugimoto, K. Araki, M. Tajima, T. Eguchi, I. Yamaga, M. Dhamrin, K. Kamisako, and T. Saitoh, J. Appl. Phys. 102, 054506 (2007).

${ }^{27}$ M. Tajima, M. Ikebe, Y. Ohshita, and A. Ogura, J. Electron. Mater. 39, 747-750 (2010)

${ }^{28}$ D. H. Macdonald, L. J. Geerligs, and A. Azzizi, J. Appl. Phys. 95, 10211028 (2004).

${ }^{29}$ T. Trupke, J. H. Zhao, A. H. Wang, R. Corkish, and M. A. Green, Appl. Phys. Lett. 82, 2996-2998 (2003).

${ }^{30}$ L. Lucy, Astron. J. 79, 745 (1974).

${ }^{31}$ W. H. Richardson, J. Opt. Soc. Am. 62, 55-59 (1972).

${ }^{32}$ T. A. Ferreira and W. Rasband, The ImageJ User Guide (National Institutes of Health, 2011).

${ }^{33}$ D. Walter, A. Liu, E. Franklin, D. Macdonald, B. Mitchell, and T. Trupke, in IEEE 38th Photovoltaic Specialists Conference, Austin, TX, 3-8 June 2012.

${ }^{34}$ D. Hinken, C. Schinke, S. Herlufsen, A. Schmidt, K. Bothe, and R. Brendel, Rev. Sci. Instrum. 82, 033706 (2011).

${ }^{35} \mathrm{~K}$. Ramspeck, K. Bothe, J. Schmidt, and R. Brendel, J. Appl. Phys. 106, 114506 (2009).

${ }^{36}$ M. C. Schubert, S. Pingel, M. The, and W. Warta, J. Appl. Phys. 101, 124907 (2007).

${ }^{37}$ M. P. Peloso, B. Hoex, and A. G. Aberle, Appl. Phys. Lett. 98, 171914 (2011).

${ }^{38}$ Y. Han, E. Choi, and M. Kang, IEEE Trans. Consum. Electron. 55, 22872293 (2009).

${ }^{39}$ T. Trupke, J. Nyhus, R. A. Sinton, and J. W. Weber, in Proceedings of the 24th European Photovoltaic Conference, Hamburg, Germany, 21-25 September 2009. 